\title{
Lyme Disease: A Bioethical Morass
}

\section{Neha Jariwala, Erum llyas and Herbert B Allen}

Department of Dermatology, Drexel University College of Medicine, 219 N. Broad St, $4^{\text {th }}$ floor, Philadelphia, United States

*Corresponding author: Herbert B Allen, Department of Dermatology, Drexel University College of Medicine, 219 N. Broad St, $4^{\text {th }}$ floor, Philadelphia, United States, Tel: 215762 5550; Fax: 215762 5570; E-mail: Herbert.Allen@drexelmed.edu

Received date: September 29, 2016; Accepted date: October 21, 2016; Published date: October 24, 2016

Copyright: $\odot 2016$ Jariwala $\mathrm{N}$ et al. This is an open-access article distributed under the terms of the Creative Commons Attribution License, which permits unrestricted use, distribution, and reproduction in any medium, provided the original author and source are credited

\begin{abstract}
"Primum non nocere", "first do no harm" is a medical dictum based in antiquity. Yet, in nearly everything related to Lyme disease, it seems almost entirely disregarded. How ethical is it that we follow the guidelines of the CDC regarding diagnosis when those guidelines require erythema migrans that is clearly recognizable only in one ("bullseye rash") of its multiple presentations? Further, how ethical is it that we are held to guidelines regarding a positive serology that is positive (at best) only $40 \%$ of the time?
\end{abstract}

Another questionable ethical situation is the use of a bacteriostatic antibiotic that barely meets the MIC for Borrelia burgdorferi in its ordinarily prescribed regimen. It is also dependent on compliance which is a huge issue because of the gastrointestinal side effects. This antibiotic may clear the rash, but seemingly does little to prevent late findings of the disease. The sub lethal antibiotic dose can be important in the subsequent development of biofilms that lead to a chronic disease state.

Lastly, how ethical is it that we have nearly abandoned our patient advocacy and permitted the insurance companies to dictate allowable treatment? And, in as much as Borrelia organisms were found in the brains of Alzheimer's disease patients over $25 \mathrm{yrs}$ ago and those spirochetes have recently been shown to produce biofilms, how ethical is it that we ignore research underpinning the pathogenesis of this disease?

The intent of this work is to discuss how all aspects of Lyme disease (LD) are bioethically challenged. We include Alzheimer's disease $(A D)$ in the discussion because Lyme spirochetes have been found in, and cultured from, the brains of $A D$. This makes $L D$, in its presentation as $A D$, the equivalent of tertiary neurosyphilis with the only difference being a different spirochete.

Keywords: Lyme disease; Spirochetes; Biofilms; Diagnosis; Serologies; Treatment

\section{Introduction}

\section{Lyme disease}

Lyme disease was named as a distinct entity in 1977. The constellations of symptoms now recognized as Lyme disease have been well described for centuries [1].

In 1909, Erythema chronicum migrans, often referred to as the target rash, was first described by Afzelius in an article published in 1921 [2]. Around this time in 1910, an expanding target rash was described as associated with an insect bite. At this point, however, it was not quite clear that the rash had any implications. From the 1930s through the late 1940s, sporadic case reports began surfacing suggesting that the expanding target rash known as erythema migrans had an association with meningitis. When Hellerstrom in 1949 confirmed a strong likelihood of the association between erythema migrans and meningitis, the medical community began to recognize the need to consider therapeutic options for this evolving entity $[3,4]$.

Lennhoff in 1948 detected spirochetal elements in a skin specimen from a patient with erythema migrans. This laid the groundwork to evaluate the effectiveness of the use of penicillin for patients with erythema migrans [4]. In 1951, successful reports of penicillin clearing the rash sooner than expected along with the resolution of neurological symptoms, even without CSF changes, continued to confirm the infectious etiology to these findings.

It was not until 1975 that the finding of oligoarthritis with erythema migrans was noted in Lyme Connecticut that the skin and joint findings were clearly linked. In 1977, Lyme disease became known as a distinct entity by describing the skin, joint, neurological and cardiac findings in a group of patients with similar findings in a similar location [5].

By 1980 , patients with the classic rash of erythema migrans were studied to determine effectiveness of tetracycline, penicillin and erythromycin in treating Lyme disease. Treatment success was determined by rash resolution and the absence of late Lyme sequelae. To determine if late sequelae were present, patients were asked to contact researchers should any neurologic or cardiac symptoms develop within a year of therapy. The absence of these symptoms as reported by patients pointed toward success of a therapeutic regimen utilizing tetracycline in dosages of a $250 \mathrm{mg}$ four times daily for 20 days (one patient on the 10-day regimen had persistence of rash) [6].

By 1982, Burgdorfer isolated the spirochete from the tick vector and also found evidence of this in the sera of patients with Lyme disease [7]. This implicated this newly discovered spirochete, Borrelia 
burgdorferi, as the causative agent in Lyme. The groundwork for antibiotic therapy in Lyme disease was laid.

Since that time, the bacteria that causes Lyme disease has been isolated from skin, blood, CSF and, most recently from brain biopsies. The hallmark of neuroborreliosis is lymphocytic pleocytosis. The actual isolation of the spirochete from the CSF is rare. The most common neurologic manifestations include Bannwarth syndrome, meningitis or mild meningoencephalitis, and late stage chronic encephalomyelitis [8-10].

The current CDC guidelines rely on the presence of erythema migrans and other constitutional symptoms such as fever, chills, headaches, joint pains, muscle pain, swollen lymph nodes and fatigue in addition to exposure to infected ticks. Two tiered laboratory testing for diagnosis of Lyme is only recommended for patients suspected to have Lyme disease [11].

\section{Alzheimers}

Alzheimer's disease was first described by Alois Alzheimer in 1908 to described the pathological changes found in the brain of a woman who died early with findings of dementia. The hallmark pathological brain findings include amyloid plaques and neurofibrillary tangles. At the time, the term Alzheimer's disease was used to refer to a subset of patients with the findings of early onset of dementia. When initially characterized it was meant to describe a specific constellation of clinical findings of memory loss and cognitive impairment noted in those younger than 60 years of age- hence it was referred to as presenile dementia. However, the most common cause of dementia was caused by the spirochetal disease known as syphilis. After the discovery of penicillin in the 1940s, the incidence of the more common form of dementia- neurosyphilis, the late stage manifestation of syphilis- dramatically declined.

After the 1960s through 1970s, Alzheimer's was increasingly recognized as more prevalent than previously thought. The pathological findings of amyloid plaques and neurofibrillary tangles that define $\mathrm{AD}$ have been noted in brains in different age groups. $20 \%$ of brains of persons who died in their 20s showed AD related changes, and $40 \%$ of cognitively normal elderly may have changes that fulfill the criteria for $\mathrm{AD}[10]$.

As $\mathrm{AD}$ continues to take shape as an entity, associations with arterial hypertension, diabetes, and hypercholesterolemia have been noted.

\section{The Controversy}

The compelling, overarching consideration in this discussion is the finding of the presence of Borrelia spirochetes in the brains of patients with Alzheimer's disease (AD). First noted on pathology by Macdonald in 1988, the presence of spirochetes was also noted by Riviere utilizing polymerase chain reaction (PCR), which identified the DNA (2002) [12,13]. Ultimately, in 2011, Miklossy confirmed the presence of spirochetes with PCR; she found dental spirochetes in $75 \%$ of the specimens and Lyme organisms in 25\% [14].

Macdonald also cultured Borrelia from an affected brain in 1988 and this was reaffirmed by Miklossy in 2016 with cultures from Alzheimer's brains that grew Borrelia [15,16]. Miklossy showed these organisms made biofilms, amyloid precursor protein, and beta amyloid in vitro. Thus, these microbes have been definitively identified in tertiary spirochetosis, yet little or no concern has been forthcoming from the medical community. In fact, these findings have been met with resounding silence for the most part. Worse in the denial of the presence of Borrelia in tertiary disease, the concept of "post treatment Lyme disease syndrome" has been promoted instead [17]. This model of pathogenesis has many proponents, and nearly all the research has been aligned with this point of view. Little attention is given to this exceedingly well documented "microbial pathogen theory" of tertiary disease.

The concept of microbial pathogenesis for Alzheimer's has briefly been addressed in the literature. Gutacker, for example, reviewed Miklossy's findings and argued that these findings were likely false positive microscopic findings of Miklossy and Macdonald in addition to potential false positive immunologic results [18]. He also pointed to Pappolla's negative results for findings spirochetes in the brains of Alzheimer's disease patients he studied. He did suggest that there may be a possible association of Borrelia in the abnormal amyloid formation such as in prion diseases. He concluded by dismissing the microbial association of Borrelia in the etiology of Alzheimer's disease by stating that if the organism has not been isolated in the brain, then it is not associated with disease. He also stated without citation that the geographical distribution of Lyme borrleiosis should parallel the geographical distribution of Alzheimer's disease.

Unfortunately, his assessment failed to acknowledge the importance of Miklossy and Macdonald's isolation of the spirochete in the brains of Alzheimer's patients - a very difficult task. By referencing another scientist's failed efforts to isolate the spirochete does not confirm false positive results. As a matter of fact, in cases of myositis associated with Lyme disease there are only rare reports of isolating the spirochete from the muscle. However, it is well established that the symptoms of myositis are a manifestation of Lyme disease [19]. Gutacker also stated that Lyme borreliosis and Alzheimer's disease do not share the same geographical distribution without referencing this assumption. LD is the most prevalent vector borne illness in the United States. It is also the most common tick borne borreliosis in North America, Europe and parts of Asia. AD is one of the most common causes of dementia.

Serology is the next consideration: at best, it is positive only $40 \%$ of the time (and this is in patients who had culture positive erythema migrans lesions) [20]. A somewhat more sensitive serologic test is considerably better, but it does not compare with the RPR for syphilis [21].

To address the question of why there is little to no serological evidence of Lyme disease in patients with Alzheimer's disease, a small study by Galbussera failed to show seropositivity for Borrelia burgdorferi in patients with $\mathrm{AD}$ [22]. The assumption here is that patients with neuroborreliosis should test positive for Lyme disease. Although it is true that for patients with long term disease to result in neuroborreliosis should presumably be more likely to have seropositivity, the reality is that the serologies used to test for late sequelae of Lyme are not ideal [23]. Of note, Galbussera did not test the CSF, only the serum.

Next to be considered are the diagnostic components of Lyme disease, long championed by the CDC as necessary elements. First is the bite of the tick; the Ixodes tick is so small that it may not be observed by the patient. Next, the erythema migrans rash may not be apparent. Or, it may not be the typical "bulls eye" presentation, but rather one of many other forms including multiple lesions or a cellulitic lesions [24]. Even to the trained observer, this diagnosis may 


\section{(10.4172/2155-9627.1000288}

Page 3 of 4

be challenging. The biopsy has a typical appearance, but is nondiagnostic.

Treatment is the last consideration. Doxycycline, the current treatment of choice, is the recommended antibiotic. It may clear the erythema migrans rash, but is clearly ineffective at preventing tertiary disease. When one compares the $35 \%$ incidence of tertiary findings seen in Lyme disease treated with doxycycline with the $35 \%$ incidence of tertiary findings in "untreated" syphilis, one sees that treating Lyme disease with doxycycline is akin to not treating it at all [24]. The reason for this may be doxycycline is bacteriostatic not bactericidal. It also is an oral medication with marked gastrointestinal side effects which dramatically affects compliance. Further, the MIC for the recommended oral dose of doxycycline is near the MIC for neuroborreliosis. This probably should be at 2 or more times the MIC to clear the pathogen effectively.

Allen has proposed treatment based on the fact that tertiary Lyme disease is the same clinically and pathologically as tertiary syphilis [25]. Neurosyphilis has disappeared over the past 40 years (except for a brief interval in the HIV epidemic) as a result of penicillin being given before the disease became evident [26] The same result could be similarly achieved in Lyme disease where penicillin is similarly effective. Just as in syphilis, this medication can be given because the pathogenic microbe has been identified and the antibiotic not only is bactericidal to all known spirochetes, but it also crosses the blood brain barrier with an effective MIC. This very likely could be done without the reliance on a large clinical trial, first because the microbes are sensitive to the antibiotic and, second, because the antibiotic is bactericidal. Also, such a trial would take many years (perhaps 30 ). More importantly, how "informed consent" could possibly be given is questionable when the result with the treatment group would be prevention of $\mathrm{AD}$, and, with the control group, would be dementia and downwardly spiraling disease.

\section{Bioethical Principles}

Lyme disease and Alzheimer's disease both pose a diagnostic dilemma. Lyme disease is hindered by correctly identifying the clinical findings that can be subtle or simply missed. A confirmatory diagnosis of Alzheimer's disease unfortunately rests on post mortem examination of the brain for the classic findings on pathology. Clinically, however, given the fact that the changes associated with Alzheimer's have been noted even in young patients without clinical evidence of disease make it all the more difficult to diagnose without serologies to help. In these cases, where a diagnostic dilemma exists, disregarding research findings is simply not rational or ethical. A more valuable approach to research and clinical practice would be to approach these dilemmas with epistemic humility. This concept is referenced by Kennedy where she argues that a "compassionate suspension of judgment" when diagnoses are difficult can only serve to further research, respect patients, and recognize that those findings that are not immediately understood will not be simply disregarded [27].

A corollary to the Lyme disease and Alzheimer's disease potential connection is found in the case of another spirochete disease, Syphilis. Neurosyphilis, also referred to as general paresis of the insane (GPI), was first described in 1809. Initially, GPI was thought to be the result of a chronic inflammatory process. When first described, the connection between syphilis and GPI was unknown. In 1857, Esmarch and Jessen suggested that syphilis and GPI may be associated based on epidemiological findings [28]. By 1905, the spirochete that causes syphilis was first isolated. This spirochete was found in the brains of GPI patients [29]. Once pencillin was discovered in 1943, the number of cases of neurosyphilis dropped dramatically, but not completely [30]. Those that already suffered the long term neurologic sequelae of neurosyphilis could not reverse the effects of the disease. These findings made a strong argument for early therapeutic intervention to avoid the long term consequences of untreated syphilis.

In 2015, Miklossy compared AD to tertiary syphilis (general paresis of the insane) and found the two disorders to be exactly the same [31]. The same plaques, the same neurofibrillary tangles, the same beta amyloid, and the same Tau protein were all present; the only difference was the presence of different spirochetes. Treponema pallidum was present in tertiary syphilis, and dental spirochetes, such as T. denticola and Lyme Borrelia were present in AD. In 2015 and 2016, Allen showed the presence of biofilms (made by the bacteria) in the same areas of hippocampal involvement as the senile plaques of $\mathrm{AD}[25,32]$. Also shown was the involvement of the innate immune system (Tolllike receptor 2 [TLR 2]) to the presence of the spirochetes and the biofilm. He showed how TLR 2 could lead to tissue destruction and to the formation of beta-amyloid (which itself is anti-microbial). Macdonald has also showed similar findings as regards biofilms and beta amyloid [33].

\section{Conclusion}

The "primum non nocere" concept taught for millennia seems to be ethically strained in all aspects of Lyme disease. The lack of attention towards peer reviewed reports and both repeated and repeatable isolation of the spirochete in the brains of $\mathrm{AD}$ patients seems to be bioethically indefensible. The fact that diagnosis of LD rests on clinically ambiguous findings that are considered essential for the diagnosis is clearly not in the best interest of the patient. In addition, to adhere to an antimicrobial treatment that is suboptimal seems hardly defensible in the context of patient care. By keeping outdated guidelines in place, insurance companies are only too willing to hold patients and physicians to these clearly inadequate diagnostic components [20]. By considering the "argument for rational research" whereby judgment is withheld until further evaluation is considered, this epistemic humility will serve research and patients far more effectively. Currently, the only FDA approved treatments for Alzheimer's disease merely address the symptoms of $\mathrm{AD}$ and not the etiology. By limiting the conversation and disregarding not only the clear presence of Borrelia in the brains of $\mathrm{AD}$ patients, but also the clear pathophysiology that has been well outlined whereby the spirochete can create biofilms well documented in the brains of $\mathrm{AD}$ patients, ignores the potential to consider early intervention in a disease that costs more than any other disease in America to treat.

\section{References}

1. Weber K, Pfister HW (1993) History of Lyme borreliosis in Europe. In: Weber K and Burgdorfer w (Edn.), Aspects of Lyme borreliosis. SpringerVerlag, Berlin, Germany, pp: 1-20.

2. Afzelius A (1921) Erythema chronicum migrans. Acta DermatoVenereologia 2: 120-125.

3. Hellerstrom S (1950) Erythema chronicum migrans Afzelius with meninigitis. South Med J 43: 330-334.

4. Lennhoff C (1948) Spirochaetes in aetiologically obscure diseases. Acta Derm Venereol 28: 295-324.

5. Steere AC, Grodzicki RL, Kornblatt AN (1983) The spirochetal etiology of Lyme disease. N Engl J Med 308: 733-740. 
Citation: Jariwala N, llyas E, Allen HB (2016) Lyme Disease: A Bioethical Morass. J Clin Res Bioeth 7: 1000288. doi: 10.4172/2155-9627.1000288

Page 4 of 4

6. Steere AC, Hutchinson GJ, Rahn DW (1983) Treatment of the early manifestations of Lyme disease. Ann Int Med 99: 22-26.

7. Burgdorfer W, Barbour AG, Hayes SF (1982) Lyme disease-a tick-borne spirochetosis? Science 216: 1317-1319.

8. Asbrink E, Hovmark A (1985) Successful cultivation of spirochetes from skin lesions of patients with erythema chronicum migrans Afzelius and acrodermatitis chronica atrophicans. Acta Pathol Microbiol Immunol Scand B 93: 161-163.

9. Preac-Mursic VB, Wilske G, Schierz HW (1984) Repeated isolation of spirochetes from the cerebrospinal fluid of a patients with meningoradiculitis Bannwarth. Eur J Clin Microbiol 3: 564-565.

10. Braak H, Braak E (1997) Frequency of stages of Alzheimer-Related Lesions in Different age categories. Neurobiology of Aging 18: 351-357.

11. Centers for Disease Control and Prevention (CDC) (1995) Recommendations for test performance and interpretation from the Second National Conference on Serologic Diagnosis of Lyme Disease. MMWR Morb Mortal Wkly Rep 44: 590-591.

12. Macdonald AB, Miranda JM (1987) Concurrent neocortical borreliosis and Alzheimer's disease. Human Pathology 18: 759-61.

13. Riviere GR, Riviere KH, Smith KS (2002) Molecular and immunological evidence of oral Treponema in the human brain and their association with Alzheimer's disease. Oral Microbiol Immunol 17: 113-118.

14. Miklossy J (2011) Alzheimer's disease - a neurospirochetosis. Analysis of the evidence following Koch's and Hill's criteria. J Neuroinflammat 8: 90.

15. Macdonald AB (1988) Concurrent neocortical borreliosis and Alzheimer's disease. Ann NY Acad Science 539: 468-470.

16. Miklossy J (2016) Bacterial Amyloid and DNA are Important Constituents of Senile Plaques: Further Evidence of the Spirochetal and Biofilm Nature of Senile Plaques. J Alz Dis 53: 1459-1473.

17. Aucott JN (2015) Post-treatment Lyme disease syndrome. Infect Dis Clin North Am 29: 309-323.

18. Gutacker M, Valsangiacomo C, Balmelli T (1998) Arguments against the involvement of Borrelia burgdorferi sensu lato in Alzheimer's disease. Res Microbiol 149: 31-37.

19. Holmgren AR, Matteson EL (2006) Lyme myositis. Arthritis Rheum 54: 2697-2700.
20. Stricker RB, Johnson L (2014) Lyme disease: Call for a "Manhattan Project" to Combat the Epidemic. PLoS Pathog 10: e1003796.

21. Nelson HD, Glass N, Huffman L, Villemyer K, Hamilton A (2004) Screening for syphilis: a brief update for the U.S. Preventive Services Task Force. Agency for Healthcare Research and Quality. Rockville, MD.

22. Galbussera A, Tremolizzo L, Isella V (2008) Lack of evidence for Borrelia burgdorferi seropositivity in Alzheimer disease. Alzheimer Dis Assoc Disord 22: 308.

23. Pomelova VG, Lorenberg EI, Kuznetsora TI (2015) C6 Peptide-Based Multiplex Phosphorescence Analysis (PHOSPHAN) for Serologic Confirmation of Lyme Borreliosis. PLOS ONE 10: e0130048.

24. Allen HB, Vin H, Warner C, Joshi S (2016) Lyme Disease: Beyond Erythema Migrans. J Clin Exp Dermatol Res 7: 330.

25. Allen HB, Morales D, Jones K, Joshi S (2016) Alzheimer's Disease: A Novel Hypothesis Integrating Spirochetes, Biofilm, and the Immune System. J Neuroinfect Dis 7: 200.

26. Pao D, Goh BT, Bingham JS (2002) Management Issues in syphilis. Drugs 62: 1447-1461.

27. Kennedy AG (2013) Differential diagnosis and the suspension of judgement. J Med Philos 38: 487-500.

28. Esmarch F, Jessen W (1857) Syphilis und Geistesstörung. Allg Zeitschr Psychiat 14: 20-32.

29. Hoffmann E (1905) Weitere Mitteilungen über das Vorkommen der Spirochäta pallida bei Syphilis. Berl Klin Wochenschr 42: 1022-1032.

30. Musher DM, Hamill RJ, Baughn RE (1990) Effects of human immunodeficiency virus (HIV) infection on the course of syphilis and on response to treatment. Ann Intern Med 113: 872-881.

31. Miklossy J (2015) Historic evidence to support a causal relationship between spirochetal infections and Alzheimer's disease. Front Aging Neurosci 7: 46.

32. Allen HB (2016) Alzheimer's disease: Assessing the role of spirochetes, biofilms, the immune system, and amyloid- $\beta$ with regard to potential treatment and prevention. J Alz Dis 53: 1271-1276.

33. https://spirodementia.wordpress.com/featured -new-discoveryboodborne-borrelia-biofilms-coated-with-beta-amyloid-7-oct-2105/ 